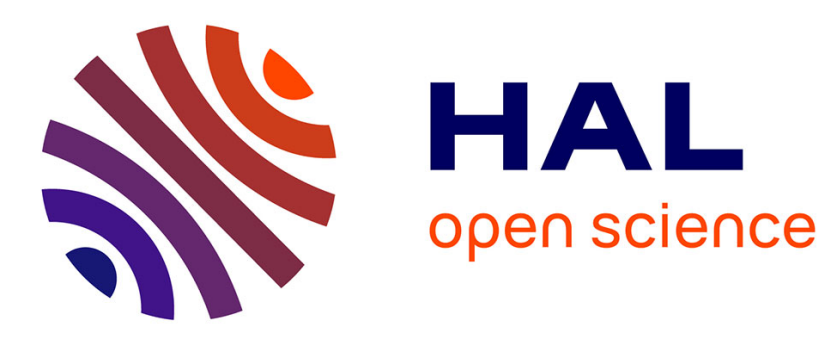

\title{
Quasi-Lovász Extensions and Their Symmetric Counterparts
}

\author{
Miguel Couceiro, Jean-Luc Marichal
}

\section{To cite this version:}

Miguel Couceiro, Jean-Luc Marichal. Quasi-Lovász Extensions and Their Symmetric Counterparts. 14th International Conference on Information Processing and Management of Uncertainty in Knowledge-Based Systems, IPMU 2012, Jul 2012, Catania, Italy. hal-01093650

\section{HAL Id: hal-01093650 https://hal.science/hal-01093650}

Submitted on 18 Feb 2017

HAL is a multi-disciplinary open access archive for the deposit and dissemination of scientific research documents, whether they are published or not. The documents may come from teaching and research institutions in France or abroad, or from public or private research centers.
L'archive ouverte pluridisciplinaire $\mathbf{H A L}$, est destinée au dépôt et à la diffusion de documents scientifiques de niveau recherche, publiés ou non, émanant des établissements d'enseignement et de recherche français ou étrangers, des laboratoires publics ou privés. 


\title{
Quasi-Lovász extensions and their symmetric counterparts
}

\author{
Miguel Couceiro and Jean-Luc Marichal \\ University of Luxembourg, Mathematics Research Unit \\ 6, rue Richard Coudenhove-Kalergi \\ L-1359 Luxembourg, G.-D. Luxembourg \\ miguel.couceiro@uni.lu, jean-luc.marichal@uni.lu
}

\begin{abstract}
We introduce the concept of quasi-Lovász extension as being a mapping $f: I^{n} \rightarrow \mathbb{R}$ defined over a nonempty real interval $I$ containing the origin, and which can be factorized as $f\left(x_{1}, \ldots, x_{n}\right)=$ $L\left(\varphi\left(x_{1}\right), \ldots, \varphi\left(x_{n}\right)\right)$, where $L$ is the Lovász extension of a pseudo-Boolean function $\psi:\{0,1\}^{n} \rightarrow \mathbb{R}$ (i.e., the function $L: \mathbb{R}^{n} \rightarrow \mathbb{R}$ whose restriction to each simplex of the standard triangulation of $[0,1]^{n}$ is the unique affine function which agrees with $\psi$ at the vertices of this simplex) and $\varphi: I \rightarrow \mathbb{R}$ is a nondecreasing function vanishing at the origin. These functions appear naturally within the scope of decision making under uncertainty since they subsume overall preference functionals associated with discrete Choquet integrals whose variables are transformed by a given utility function.

To axiomatize the class of quasi-Lovász extensions, we propose generalizations of properties used to characterize the Lovász extensions, including a comonotonic version of modularity and a natural relaxation of homogeneity. A variant of the latter property enables us to axiomatize also the class of symmetric quasi-Lovász extensions, which are compositions of symmetric Lovász extensions with 1-place nondecreasing odd functions.
\end{abstract}

Keywords: Aggregation function, discrete Choquet integral, Lovász extension, comonotonic modularity, invariance under horizontal differences.

MSC classes: 39B22, 39B72 (Primary) 26B35 (Secondary)

\section{Introduction}

Aggregation functions arise wherever merging information is needed: applied and pure mathematics (probability, statistics, decision theory, functional equations), operations research, computer science, and many applied fields (economics and finance, pattern recognition and image processing, data fusion, etc.). For recent references, see Beliakov et al. [1] and Grabisch et al. [7].

The discrete Choquet integral has been widely investigated in aggregation theory due to its many applications, for instance, in decision making (see the 
edited book [8]). A convenient way to introduce the discrete Choquet integral is via the concept of Lovász extension. An $n$-place Lovász extension is a continuous function $L: \mathbb{R}^{n} \rightarrow \mathbb{R}$ whose restriction to each of the $n$ ! subdomains

$$
\mathbb{R}_{\sigma}^{n}=\left\{\mathbf{x}=\left(x_{1}, \ldots, x_{n}\right) \in \mathbb{R}^{n}: x_{\sigma(1)} \leqslant \cdots \leqslant x_{\sigma(n)}\right\}, \quad \sigma \in S_{n},
$$

is an affine function, where $S_{n}$ denotes the set of permutations on $[n]=\{1, \ldots, n\}$. An $n$-place Choquet integral is simply a nondecreasing (in each variable) $n$-place Lovász extension which vanishes at the origin. For general background, see [7, $\S 5.4]$.

The class of $n$-place Lovász extensions has been axiomatized by the authors [4] by means of two noteworthy aggregation properties, namely comonotonic additivity and horizontal min-additivity (for earlier axiomatizations of the $n$ place Choquet integrals, see, e.g., $[2,6])$. Recall that a function $f: \mathbb{R}^{n} \rightarrow \mathbb{R}$ is said to be comonotonically additive if, for every $\sigma \in S_{n}$, we have

$$
f\left(\mathbf{x}+\mathbf{x}^{\prime}\right)=f(\mathbf{x})+f\left(\mathbf{x}^{\prime}\right), \quad \mathbf{x}, \mathbf{x}^{\prime} \in \mathbb{R}_{\sigma}^{n} .
$$

The function $f$ is said to be horizontally min-additive if

$$
f(\mathbf{x})=f(\mathbf{x} \wedge c)+f(\mathbf{x}-(\mathbf{x} \wedge c)), \quad \mathbf{x} \in \mathbb{R}^{n}, c \in \mathbb{R},
$$

where $\mathbf{x} \wedge c$ denotes the $n$-tuple whose $i$ th component is $x_{i} \wedge c=\min \left(x_{i}, c\right)$.

In this paper we consider a generalization of Lovász extensions, which we call quasi-Lovász extensions, and which are best described by the following equation

$$
f\left(x_{1}, \ldots, x_{n}\right)=L\left(\varphi\left(x_{1}\right), \ldots, \varphi\left(x_{n}\right)\right)
$$

where $L$ is a Lovász extension and $\varphi$ a nondecreasing function such that $\varphi(0)=0$. Such an aggregation function is used in decision under uncertainty, where $\varphi$ is a utility function and $f$ an overall preference functional. It is also used in multicriteria decision making where the criteria are commensurate (i.e., expressed in a common scale). For a recent reference, see Bouyssou et al. [3].

To axiomatize the class of quasi-Lovász extensions, we propose the following generalizations of comonotonic additivity and horizontal min-additivity, namely comonotonic modularity and invariance under horizontal min-differences (as well as its dual counterpart), which we now briefly describe. We say that a function $f: \mathbb{R}^{n} \rightarrow \mathbb{R}$ is comonotonically modular if, for every $\sigma \in S_{n}$, we have

$$
f(\mathbf{x})+f\left(\mathbf{x}^{\prime}\right)=f\left(\mathbf{x} \wedge \mathbf{x}^{\prime}\right)+f\left(\mathbf{x} \vee \mathbf{x}^{\prime}\right), \quad \mathbf{x}, \mathbf{x}^{\prime} \in \mathbb{R}_{\sigma}^{n},
$$

where $\mathbf{x} \wedge \mathbf{x}^{\prime}$ (resp. $\left.\mathbf{x} \vee \mathbf{x}^{\prime}\right)$ denotes the $n$-tuple whose $i$ th component is $x_{i} \wedge$ $x_{i}^{\prime}=\min \left(x_{i}, x_{i}^{\prime}\right)\left(\right.$ resp. $\left.x_{i} \vee x_{i}^{\prime}=\max \left(x_{i}, x_{i}^{\prime}\right)\right)$. We say that $f$ is invariant under horizontal min-differences if

$$
f(\mathbf{x})-f(\mathbf{x} \wedge c)=f\left([\mathbf{x}]_{c}\right)-f\left([\mathbf{x}]_{c} \wedge c\right), \quad \mathbf{x} \in \mathbb{R}^{n}, c \in \mathbb{R},
$$

where $[\mathbf{x}]_{c}$ denotes the $n$-tuple whose $i$ th component is 0 , if $x_{i} \leqslant c$, and $x_{i}$, otherwise. 
The outline of this paper is as follows. In Section 2 we recall the definitions of Lovász extensions, discrete Choquet integrals, as well as their symmetric versions, and present representations for these functions. In Section 3 we define the concept of quasi-Lovász extension and its symmetric version, introduce natural relaxations of homogeneity, namely weak homogeneity and odd homogeneity, and present characterizations of those quasi-Lovász extensions (resp. symmetric quasi-Lovász extensions) that are weakly homogeneous (resp. oddly homogeneous). In Section 4 we define the concepts of comonotonic modularity, invariance under horizontal min-differences and invariance under horizontal max-differences, and present a complete description of those function classes axiomatized by each of these properties. In Section 5 we give axiomatizations of the class of quasi-Lovász extensions by means of the properties above, and give all possible factorizations of quasi-Lovász extensions into compositions of Lovász extensions with 1-place functions. Finally, in Section 6 we present analogous results for the symmetric quasi-Lovász extensions.

We employ the following notation throughout the paper. Let $\mathbb{B}=\{0,1\}$, $\mathbb{R}_{+}=\left[0,+\infty\left[\right.\right.$, and $\left.\left.\mathbb{R}_{-}=\right]-\infty, 0\right]$. The symbol $I$ denotes a nonempty real interval, possibly unbounded, containing 0 . We also introduce the notation $I_{+}=$ $I \cap \mathbb{R}_{+}, I_{-}=I \cap \mathbb{R}_{-}$, and $I_{\sigma}^{n}=I^{n} \cap \mathbb{R}_{\sigma}^{n}$. A function $f: I^{n} \rightarrow \mathbb{R}$, where $I$ is centered at 0 , is said to be odd if $f(-\mathbf{x})=-f(\mathbf{x})$. For any function $f: I^{n} \rightarrow \mathbb{R}$, we define $f_{0}=f-f(\mathbf{0})$. For every $A \subseteq[n]$, the symbol $\mathbf{1}_{A}$ denotes the $n$-tuple whose $i$ th component is 1 , if $i \in A$, and 0 , otherwise. Let also $\mathbf{1}=\mathbf{1}_{[n]}$ and $\mathbf{0}=\mathbf{1}_{\varnothing}$. The symbols $\wedge$ and $\vee$ denote the minimum and maximum functions, respectively. For every $\mathbf{x} \in \mathbb{R}^{n}$, let $\mathbf{x}^{+}=\mathbf{x} \vee 0$ and $\mathbf{x}^{-}=(-\mathbf{x})^{+}$. For every $\mathbf{x} \in \mathbb{R}^{n}$ and every $c \in \mathbb{R}_{+}$(resp. $c \in \mathbb{R}_{-}$) we denote by $[\mathbf{x}]_{c}$ (resp. $\left.[\mathbf{x}]^{c}\right)$ the $n$-tuple whose $i$ th component is 0 , if $x_{i} \leqslant c$ (resp. $x_{i} \geqslant c$ ), and $x_{i}$, otherwise.

In order not to restrict our framework to functions defined on $\mathbb{R}$, we consider functions defined on intervals $I$ containing 0 , in particular of the forms $I_{+}, I_{-}$, and those centered at 0 .

A full version of the current paper appeared as [5].

\section{Lovász extensions and symmetric Lovász extensions}

We now recall the concepts of Lovász extension and symmetric Lovász extension.

Consider an $n$-place pseudo-Boolean function, i.e. a function $\psi: \mathbb{B}^{n} \rightarrow \mathbb{R}$, and define the set function $v_{\psi}: 2^{[n]} \rightarrow \mathbb{R}$ by $v_{\psi}(A)=\psi\left(\mathbf{1}_{A}\right)$ for every $A \subseteq[n]$. Hammer and Rudeanu [9] showed that such a function has a unique representation as a multilinear polynomial of $n$ variables

$$
\psi(\mathbf{x})=\sum_{A \subseteq[n]} a_{\psi}(A) \prod_{i \in A} x_{i},
$$

where the set function $a_{\psi}: 2^{[n]} \rightarrow \mathbb{R}$, called the Möbius transform of $v_{\psi}$, is defined by

$$
a_{\psi}(A)=\sum_{B \subseteq A}(-1)^{|A|-|B|} v_{\psi}(B) .
$$


The Lovász extension of a pseudo-Boolean function $\psi: \mathbb{B}^{n} \rightarrow \mathbb{R}$ is the function $L_{\psi}: \mathbb{R}^{n} \rightarrow \mathbb{R}$ whose restriction to each subdomain $\mathbb{R}_{\sigma}^{n}\left(\sigma \in S_{n}\right)$ is the unique affine function which agrees with $\psi$ at the $n+1$ vertices of the $n$-simplex $[0,1]^{n} \cap \mathbb{R}_{\sigma}^{n}$ (see $\left.[10,12]\right)$. We then have $\left.L_{\psi}\right|_{\mathbb{B}^{n}}=\psi$.

It can be shown (see $[7, \S 5.4 .2]$ ) that the Lovász extension of a pseudo-Boolean function $\psi: \mathbb{B}^{n} \rightarrow \mathbb{R}$ is the continuous function

$$
L_{\psi}(\mathbf{x})=\sum_{A \subseteq[n]} a_{\psi}(A) \bigwedge_{i \in A} x_{i}, \quad \mathbf{x} \in \mathbb{R}^{n}
$$

Its restriction to $\mathbb{R}_{\sigma}^{n}$ is the affine function

$$
L_{\psi}(\mathbf{x})=\psi(\mathbf{0})+\sum_{i \in[n]} x_{\sigma(i)}\left(v_{\psi}\left(A_{\sigma}^{\uparrow}(i)\right)-v_{\psi}\left(A_{\sigma}^{\uparrow}(i+1)\right)\right), \quad \mathbf{x} \in \mathbb{R}_{\sigma}^{n},
$$

or equivalently,

$$
L_{\psi}(\mathbf{x})=\psi(\mathbf{0})+\sum_{i \in[n]} x_{\sigma(i)}\left(L_{\psi}\left(\mathbf{1}_{A_{\sigma}^{\uparrow}(i)}\right)-L_{\psi}\left(\mathbf{1}_{A_{\sigma}^{\uparrow}(i+1)}\right)\right), \quad \mathbf{x} \in \mathbb{R}_{\sigma}^{n},
$$

where $A_{\sigma}^{\uparrow}(i)=\{\sigma(i), \ldots, \sigma(n)\}$, with the convention that $A_{\sigma}^{\uparrow}(n+1)=\varnothing$. Indeed, for any $k \in[n+1]$, both sides of each of the equations (1) and (2) agree at $\mathbf{x}=\mathbf{1}_{A_{\sigma}^{\uparrow}(k)}$. It is noteworthy that $L_{\psi}$ can also be represented by

$$
L_{\psi}(\mathbf{x})=\psi(\mathbf{0})+\sum_{i \in[n]} x_{\sigma(i)}\left(L_{\psi}\left(-\mathbf{1}_{A_{\sigma}^{\downarrow}(i-1)}\right)-L_{\psi}\left(-\mathbf{1}_{A_{\sigma}^{\downarrow}(i)}\right)\right), \quad \mathbf{x} \in \mathbb{R}_{\sigma}^{n},
$$

where $A_{\sigma}^{\downarrow}(i)=\{\sigma(1), \ldots, \sigma(i)\}$, with the convention that $A_{\sigma}^{\downarrow}(0)=\varnothing$. Indeed, for any $k \in[n+1]$, by $(2)$ we have $L_{\psi}\left(-\mathbf{1}_{A_{\sigma}^{\downarrow}(k-1)}\right)=\psi(\mathbf{0})+L_{\psi}\left(\mathbf{1}_{A_{\sigma}^{\uparrow}(k)}\right)-L_{\psi}\left(\mathbf{1}_{A_{\sigma}^{\uparrow}(1)}\right)$.

Let $\psi^{d}$ denote the dual of $\psi$, that is the function $\psi^{d}: \mathbb{B}^{n} \rightarrow \mathbb{R}$ defined by $\psi^{d}(\mathbf{x})=\psi(\mathbf{0})+\psi(\mathbf{1})-\psi(\mathbf{1}-\mathbf{x})$. The next result provides further representations for $L_{\psi}$.

Proposition 1. The Lovász extension of a pseudo-Boolean function $\psi: \mathbb{B}^{n} \rightarrow$ $\mathbb{R}$ is given by

$$
L_{\psi}(\mathbf{x})=\psi(\mathbf{0})+\sum_{A \subseteq[n]} a_{\psi^{d}}(A) \bigvee_{i \in A} x_{i}
$$

and

$$
L_{\psi}(\mathbf{x})=\psi(\mathbf{0})+L_{\psi}\left(\mathbf{x}^{+}\right)-L_{\psi^{d}}\left(\mathbf{x}^{-}\right) .
$$

A function $f: \mathbb{R}^{n} \rightarrow \mathbb{R}$ is said to be a Lovász extension if there is a pseudoBoolean function $\psi: \mathbb{B}^{n} \rightarrow \mathbb{R}$ such that $f=L_{\psi}$.

An $n$-place Choquet integral is a nondecreasing Lovász extension $L_{\psi}: \mathbb{R}^{n} \rightarrow$ $\mathbb{R}$ such that $L_{\psi}(\mathbf{0})=0$. It is easy to see that a Lovász extension $L: \mathbb{R}^{n} \rightarrow \mathbb{R}$ is an $n$-place Choquet integral if and only if its underlying pseudo-Boolean function $\psi=\left.L\right|_{\mathbb{B}^{n}}$ is nondecreasing and vanishes at the origin (see $[7, \S 5.4]$ ). 
The symmetric Lovász extension of a pseudo-Boolean function $\psi: \mathbb{B}^{n} \rightarrow \mathbb{R}$ is the function $\check{L}: \mathbb{R}^{n} \rightarrow \mathbb{R}$ defined by $\breve{L}_{\psi}(\mathbf{x})=\psi(\mathbf{0})+L_{\psi}\left(\mathbf{x}^{+}\right)-L_{\psi}\left(\mathbf{x}^{-}\right)$(see [4]). In particular, we see that $\check{L}_{\psi}-\check{L}_{\psi}(\mathbf{0})=\breve{L}_{\psi}-\psi(\mathbf{0})$ is an odd function.

It is easy to see that the restriction of $\check{L}_{\psi}$ to $\mathbb{R}_{\sigma}^{n}$ is the function

$$
\begin{aligned}
\check{L}_{\psi}(\mathbf{x})= & \psi(\mathbf{0})+\sum_{1 \leqslant i \leqslant p} x_{\sigma(i)}\left(L_{\psi}\left(\mathbf{1}_{A_{\sigma}^{\downarrow}(i)}\right)-L_{\psi}\left(\mathbf{1}_{A_{\sigma}^{\downarrow}(i-1)}\right)\right) \\
& +\sum_{p+1 \leqslant i \leqslant n} x_{\sigma(i)}\left(L_{\psi}\left(\mathbf{1}_{A_{\sigma}^{\uparrow}(i)}\right)-L_{\psi}\left(\mathbf{1}_{A_{\sigma}^{\uparrow}(i+1)}\right)\right), \quad \mathbf{x} \in \mathbb{R}_{\sigma}^{n},
\end{aligned}
$$

where the integer $p \in\{0, \ldots, n\}$ is such that $x_{\sigma(p)}<0 \leqslant x_{\sigma(p+1)}$.

A function $f: \mathbb{R}^{n} \rightarrow \mathbb{R}$ is said to be a symmetric Lovász extension if there is a pseudo-Boolean function $\psi: \mathbb{B}^{n} \rightarrow \mathbb{R}$ such that $f=\check{L}_{\psi}$. Nondecreasing symmetric Lovász extensions vanishing at the origin, also called discrete symmetric Choquet integrals, were introduced by Šipoš [13] (see also [7, §5.4]).

\section{Quasi-Lovász extensions and symmetric quasi-Lovász extensions}

In this section we introduce the concepts of quasi-Lovász extension and symmetric quasi-Lovász extension. We also introduce natural relaxations of homogeneity, namely weak homogeneity and odd homogeneity, and present a characterization of those quasi-Lovász extensions (resp. symmetric quasi-Lovász extensions) that are weakly homogeneous (resp. oddly homogeneous). Recall that $I$ is a real interval containing 0 .

A quasi-Lovász extension is a function $f: I^{n} \rightarrow \mathbb{R}$ defined by

$$
f=L \circ(\varphi, \ldots, \varphi)
$$

also written $f=L \circ \varphi$, where $L: \mathbb{R}^{n} \rightarrow \mathbb{R}$ is a Lovász extension and $\varphi: I \rightarrow \mathbb{R}$ is a nondecreasing function satisfying $\varphi(0)=0$. Observe that a function $f: I^{n} \rightarrow \mathbb{R}$ is a quasi-Lovász extension if and only if $f_{0}=L_{0} \circ \varphi$, where $f_{0}=f-f(\mathbf{0})$ and $L_{0}=L-L(\mathbf{0})$.

Lemma 2. Assume $I \subseteq \mathbb{R}_{+}$. For every quasi-Lovász extension $f: I^{n} \rightarrow \mathbb{R}$, $f=L \circ \varphi$, we have

$$
f_{0}\left(x \mathbf{1}_{A}\right)=\varphi(x) L_{0}\left(\mathbf{1}_{A}\right), \quad x \in I, A \subseteq[n] .
$$

Observe that if $[0,1] \subseteq I \subseteq \mathbb{R}_{+}$and $\varphi(1)=1$, then the equation in $(3)$ becomes $f_{0}\left(x \mathbf{1}_{A}\right)=\varphi(x) f_{0}\left(\mathbf{1}_{A}\right)$. This motivates the following definition. We say that a function $f: I^{n} \rightarrow \mathbb{R}$, where $I \subseteq \mathbb{R}_{+}$, is weakly homogeneous if there exists a nondecreasing function $\varphi: I \rightarrow \mathbb{R}$ satisfying $\varphi(0)=0$ such that $f\left(x \mathbf{1}_{A}\right)=$ $\varphi(x) f\left(\mathbf{1}_{A}\right)$ for every $x \in I$ and every $A \subseteq[n]$. Clearly, every weakly homogeneous function $f$ satisfies $f(\mathbf{0})=0$ (take $x=0$ in the definition). 
Proposition 3. Assume $[0,1] \subseteq I \subseteq \mathbb{R}_{+}$. Let $f: I^{n} \rightarrow \mathbb{R}$ be a nonconstant quasi-Lovász extension, $f=L \circ \varphi$. Then the following are equivalent.

(i) $f_{0}$ is weakly homogeneous.

(ii) There exists $A \subseteq[n]$ such that $f_{0}\left(\mathbf{1}_{A}\right) \neq 0$.

(iii) $\varphi(1) \neq 0$.

In this case we have $f_{0}\left(x \mathbf{1}_{A}\right)=\frac{\varphi(x)}{\varphi(1)} f_{0}\left(\mathbf{1}_{A}\right)$ for every $x \in I$ and every $A \subseteq[n]$.

Remark 4. (a) If $[0,1] \varsubsetneqq I \subseteq \mathbb{R}_{+}$, then the quasi-Lovász extension $f: I^{n} \rightarrow \mathbb{R}$ defined by $f(\mathbf{x})=\bigwedge_{i \in[n]} \varphi\left(x_{i}\right)$, where $\varphi(x)=0 \vee(x-1)$, is not weakly homogeneous.

(b) When $I=[0,1]$, the assumption that $f$ is nonconstant implies immediately that $\varphi(1) \neq 0$. We then see by Proposition 3 that $f_{0}$ is weakly homogeneous. Note also that, if $f$ is constant, then $f_{0} \equiv 0$ is clearly weakly homogeneous. Thus, for any quasi-Lovász extension $f:[0,1]^{n} \rightarrow \mathbb{R}$, the function $f_{0}$ is weakly homogeneous.

Assume now that $-x \in I$ whenever $x \in I$, that is, $I$ is centered at 0 . A symmetric quasi-Lovász extension is a function $f: I^{n} \rightarrow \mathbb{R}$ defined by $f=$ $\breve{L} \circ \varphi$, where $\check{L}: \mathbb{R}^{n} \rightarrow \mathbb{R}$ is a symmetric Lovász extension and $\varphi: I \rightarrow \mathbb{R}$ is a nondecreasing odd function.

We say that a function $f: I^{n} \rightarrow \mathbb{R}$, where $I$ centered at 0 , is oddly homogeneous if there exists a nondecreasing odd function $\varphi: I \rightarrow \mathbb{R}$ such that $f\left(x \mathbf{1}_{A}\right)=\varphi(x) f\left(\mathbf{1}_{A}\right)$ for every $x \in I$ and every $A \subseteq[n]$. Clearly, for every oddly homogeneous function $f$, the functions $\left.f\right|_{I_{+}^{n}}$ and $\left.f\right|_{I_{-}^{n}}$ are weakly homogeneous.

Proposition 5. Assume that $I$ is centered at 0 with $[-1,1] \subseteq I$. Let $f: I^{n} \rightarrow \mathbb{R}$ be a symmetric quasi-Lovász extension, $f=\check{L} \circ \varphi$, such that $\left.f\right|_{I_{+}^{n}}$ or $\left.f\right|_{I_{-}^{n}}$ is nonconstant. Then the following are equivalent.

(i) $f_{0}$ is oddly homogeneous.

(ii) There exists $A \subseteq[n]$ such that $f_{0}\left(\mathbf{1}_{A}\right) \neq 0$.

(iii) $\varphi(1) \neq 0$.

In this case we have $f_{0}\left(x \mathbf{1}_{A}\right)=\frac{\varphi(x)}{\varphi(1)} f_{0}\left(\mathbf{1}_{A}\right)$ for every $x \in I$ and every $A \subseteq[n]$.

Remark 6. Similarly to Remark 4(b), we see that, for any symmetric quasiLovász extension $f:[-1,1]^{n} \rightarrow \mathbb{R}$, the function $f_{0}$ is oddly homogeneous.

\section{Comonotonic modularity}

Recall that a function $f: I^{n} \rightarrow \mathbb{R}$ is said to be modular (or a valuation) if

$$
f(\mathbf{x})+f\left(\mathbf{x}^{\prime}\right)=f\left(\mathbf{x} \wedge \mathbf{x}^{\prime}\right)+f\left(\mathbf{x} \vee \mathbf{x}^{\prime}\right)
$$

for every $\mathbf{x}, \mathbf{x}^{\prime} \in I^{n}$. It was proved (see Topkis [14, Thm 3.3]) that a function $f: I^{n} \rightarrow \mathbb{R}$ is modular if and only if it is separable, that is, there exist $n$ functions 
$f_{i}: I \rightarrow \mathbb{R}, i \in[n]$, such that $f=\sum_{i \in[n]} f_{i}$. In particular, any 1-place function $f: I \rightarrow \mathbb{R}$ is modular.

Two $n$-tuples $\mathbf{x}, \mathbf{x}^{\prime} \in I^{n}$ are said to be comonotonic if there exists $\sigma \in S_{n}$ such that $\mathbf{x}, \mathbf{x}^{\prime} \in I_{\sigma}^{n}$. A function $f: I^{n} \rightarrow \mathbb{R}$ is said to be comonotonically modular (or a comonotonic valuation) if (4) holds for every comonotonic $n$-tuples $\mathbf{x}, \mathbf{x}^{\prime} \in I^{n}$. This notion was considered in the special case when $I=[0,1]$ in [11]. We observe that, for any function $f: I^{n} \rightarrow \mathbb{R}$, condition (4) holds for every $\mathbf{x}, \mathbf{x}^{\prime} \in I^{n}$ of the forms $\mathbf{x}=x \mathbf{1}_{A}$ and $\mathbf{x}^{\prime}=x^{\prime} \mathbf{1}_{A}$, where $x, x^{\prime} \in I$ and $A \subseteq[n]$. Observe also that, for every $\mathbf{x} \in \mathbb{R}_{+}^{n}$ and every $c \in \mathbb{R}_{+}$, we have $\mathbf{x}-\mathbf{x} \wedge c=[\mathbf{x}]_{c}-[\mathbf{x}]_{c} \wedge c$. This motivates the following definition. We say that a function $f: I^{n} \rightarrow \mathbb{R}$, where $I \subseteq \mathbb{R}_{+}$, is invariant under horizontal min-differences if, for every $\mathbf{x} \in I^{n}$ and every $c \in I$, we have

$$
f(\mathbf{x})-f(\mathbf{x} \wedge c)=f\left([\mathbf{x}]_{c}\right)-f\left([\mathbf{x}]_{c} \wedge c\right) .
$$

Dually, we say that a function $f: I^{n} \rightarrow \mathbb{R}$, where $I \subseteq \mathbb{R}_{-}$, is invariant under horizontal max-differences if, for every $\mathbf{x} \in I^{n}$ and every $c \in I$, we have

$$
f(\mathbf{x})-f(\mathbf{x} \vee c)=f\left([\mathbf{x}]^{c}\right)-f\left([\mathbf{x}]^{c} \vee c\right) .
$$

We observe that, for any function $f: I^{n} \rightarrow \mathbb{R}$, where $I \subseteq \mathbb{R}_{+}$, condition (5) holds for every $\mathbf{x} \in I^{n}$ of the form $\mathbf{x}=x \mathbf{1}_{A}$, where $x \in I$ and $A \subseteq[n]$. Dually, for any function $f: I^{n} \rightarrow \mathbb{R}$, where $I \subseteq \mathbb{R}_{-}$, condition (6) holds for every tuple $\mathbf{x} \in I^{n}$ of the form $\mathbf{x}=x \mathbf{1}_{A}$, where $x \in I$ and $A \subseteq[n]$. We also observe that a function $f$ is comonotonically modular (resp. invariant under horizontal mindifferences, invariant under horizontal max-differences) if and only if so is the function $f_{0}$.

Theorem 7. Assume $I \subseteq \mathbb{R}_{+}$and let $f: I^{n} \rightarrow \mathbb{R}$ be a function. Then the following are equivalent.

(i) $f$ is comonotonically modular.

(ii) $f$ is invariant under horizontal min-differences.

(iii) There exists a function $g: I^{n} \rightarrow \mathbb{R}$ such that, for every $\sigma \in S_{n}$ and every $\mathbf{x} \in I_{\sigma}^{n}$, we have

$$
f(\mathbf{x})=g(\mathbf{0})+\sum_{i \in[n]}\left(g\left(x_{\sigma(i)} \mathbf{1}_{A_{\sigma}^{\uparrow}(i)}\right)-g\left(x_{\sigma(i)} \mathbf{1}_{A_{\sigma}^{\uparrow}(i+1)}\right)\right) .
$$

In this case, we can choose $g=f$.

Remark 8. The equivalence between $(i)$ and (iii) in Theorem 7 generalizes Theorem 1 in [11], which describes the class of comonotonically modular functions $f:[0,1]^{n} \rightarrow[0,1]$ under the additional conditions of symmetry and idempotence.

We observe that if $f: I^{n} \rightarrow \mathbb{R}$ is comonotonically modular then necessarily

$$
f_{0}(\mathbf{x})=f_{0}\left(\mathbf{x}^{+}\right)+f_{0}\left(-\mathbf{x}^{-}\right) \quad\left(\text { take } \mathbf{x}^{\prime}=\mathbf{0} \text { in }(4)\right) .
$$

We may now present a characterization of the class of comonotonically modular functions on an arbitrary interval $I$ containing 0 . 
Theorem 9. For any function $f: I^{n} \rightarrow \mathbb{R}$, the following are equivalent.

(i) $f$ is comonotonically modular.

(ii) There exist $g: I_{+}^{n} \rightarrow \mathbb{R}$ comonotonically modular (or invariant under horizontal min-differences) and $h: I_{-}^{n} \rightarrow \mathbb{R}$ comonotonically modular (or invariant under horizontal max-differences) such that $f_{0}(\mathbf{x})=g_{0}\left(\mathbf{x}^{+}\right)+h_{0}\left(-\mathbf{x}^{-}\right)$ for every $\mathbf{x} \in I^{n}$. In this case, we can choose $g=\left.f\right|_{I_{+}^{n}}$ and $h=\left.f\right|_{I_{-}^{n}}$.

(iii) There exist $g: I_{+}^{n} \rightarrow \mathbb{R}$ and $h: I_{-}^{n} \rightarrow \mathbb{R}$ such that, for every $\sigma \in S_{n}$ and every $\mathbf{x} \in I_{\sigma}^{n}$,

$$
\begin{aligned}
f_{0}(\mathbf{x})= & \sum_{1 \leqslant i \leqslant p}\left(h\left(x_{\sigma(i)} \mathbf{1}_{A_{\sigma}^{\downarrow}(i)}\right)-h\left(x_{\sigma(i)} \mathbf{1}_{A_{\sigma}^{\downarrow}(i-1)}\right)\right) \\
& +\sum_{p+1 \leqslant i \leqslant n}\left(g\left(x_{\sigma(i)} \mathbf{1}_{A_{\sigma}^{\uparrow}(i)}\right)-g\left(x_{\sigma(i)} \mathbf{1}_{A_{\sigma}^{\uparrow}(i+1)}\right)\right),
\end{aligned}
$$

where $p \in\{0, \ldots, n\}$ is such that $x_{\sigma(p)}<0 \leqslant x_{\sigma(p+1)}$. In this case, we can choose $g=\left.f\right|_{I_{+}^{n}}$ and $h=\left.f\right|_{I_{-}^{n}}$.

Remark 10. Observe that using condition (iii) in Theorems 7 and 9, we can easily derive characterizations of Choquet integrals and of symmetric Choquet integrals given in terms of comonotonic modularity. Indeed, we simply need to suppose that $f: I^{n} \rightarrow \mathbb{R}$ is nondecreasing and satisfies

$$
f\left(x \mathbf{1}_{A}\right)=x f\left(\mathbf{1}_{A}\right), \quad \text { for every } x \in I \text { and every } A \subseteq[n],
$$

and assume that $[0,1] \subseteq I \subseteq \mathbb{R}_{+}$(in Theorem 7) and that $I$ is centered at 0 with $[-1,1] \subseteq I \subseteq \mathbb{R}$ (in Theorem 9).

From Theorem 9 we obtain the "comonotonic" analogue of Topkis' characterization [14] of modular functions as separable functions, and which provides an alternative description of comonotonically modular functions.

Corollary 11. Let $J$ be any nonempty real interval, possibly unbounded. A function $f: J^{n} \rightarrow \mathbb{R}$ is comonotonically modular if and only if it is comonotonically separable, that is, for every $\sigma \in S_{n}$, there exist functions $f_{i}^{\sigma}: J \rightarrow \mathbb{R}, i \in[n]$, such that

$$
f(\mathbf{x})=\sum_{i=1}^{n} f_{i}^{\sigma}\left(x_{\sigma(i)}\right)=\sum_{i=1}^{n} f_{\sigma^{-1}(i)}^{\sigma}\left(x_{i}\right), \quad \mathbf{x} \in J^{n} \cap \mathbb{R}_{\sigma}^{n} .
$$

\section{Axiomatization and representation of quasi-Lovász extensions and their symmetric counterparts}

We now present axiomatizations of the class of quasi-Lovász extensions and describe all possible factorizations of quasi-Lovász extensions into compositions of Lovász extensions with 1-place nondecreasing functions. Similarly, we provide analogous results concerning the class of symmetric quasi-Lovász extensions. 
Theorem 12. Assume $[0,1] \subseteq I \subseteq \mathbb{R}_{+}$and let $f: I^{n} \rightarrow \mathbb{R}$ be a nonconstant function. Then the following are equivalent.

(i) $f$ is a quasi-Lovász extension and there exists $A \subseteq[n]$ such that $f_{0}\left(\mathbf{1}_{A}\right) \neq 0$.

(ii) $f$ is comonotonically modular (or invariant under horizontal min-differences) and $f_{0}$ is weakly homogeneous.

(iii) There is a nondecreasing function $\varphi_{f}: I \rightarrow \mathbb{R}$ satisfying $\varphi_{f}(0)=0$ and $\varphi_{f}(1)=1$ such that $f=L_{\left.f\right|_{\mathbb{B}}} \circ \varphi_{f}$.

Let $f: I^{n} \rightarrow \mathbb{R}$ be a quasi-Lovász extension, where $[0,1] \subseteq I \subseteq \mathbb{R}_{+}$, for which there exists $A^{*} \subseteq[n]$ such that $f_{0}\left(\mathbf{1}_{A^{*}}\right) \neq 0$. Then the inner function $\varphi_{f}$ introduced in Theorem 12 is unique. Indeed, by Proposition 3 , we have $f_{0}\left(x \mathbf{1}_{A}\right)=$ $\varphi_{f}(x) f_{0}\left(\mathbf{1}_{A}\right)$ for every $x \in I$ and every $A \subseteq[n]$. The function $\varphi_{f}$ is then defined by $\varphi_{f}(x)=\frac{f_{0}\left(x \mathbf{1}_{A^{*}}\right)}{f_{0}\left(\mathbf{1}_{A^{*}}\right)}, x \in I$.

Theorem 13. Assume $[0,1] \subseteq I \subseteq \mathbb{R}_{+}$and let $f: I^{n} \rightarrow \mathbb{R}$ be a quasi-Lovász extension, $f=L \circ \varphi$. Then there exists $A^{*} \subseteq[n]$ such that $f_{0}\left(\mathbf{1}_{A^{*}}\right) \neq 0$ if and only if there exists $a>0$ such that $\varphi=a \varphi_{f}$ and $L_{0}=\frac{1}{a}\left(L_{\left.f\right|_{\mathbb{B}}}\right)_{0}$.

We now present an axiomatization of the class of symmetric quasi-Lovász extensions and describe all possible factorizations of symmetric quasi-Lovász extensions into compositions of symmetric Lovász extensions with 1-place nondecreasing odd functions.

Theorem 14. Assume that $I$ is centered at 0 with $[-1,1] \subseteq I$ and let $f: I^{n} \rightarrow$ $\mathbb{R}$ be a function such that $\left.f\right|_{I_{+}^{n}}$ or $\left.f\right|_{I_{-}^{n}}$ is nonconstant. Then the following are equivalent.

(i) $f$ is a symmetric quasi-Lovász extension and there exists $A \subseteq[n]$ such that $f_{0}\left(\mathbf{1}_{A}\right) \neq 0$.

(ii) $f$ is comonotonically modular and $f_{0}$ is oddly homogeneous.

(iii) There is a nondecreasing odd function $\varphi_{f}: I \rightarrow \mathbb{R}$ satisfying $\varphi_{f}(1)=1$ such that $f=\check{L}_{f \mid \mathbb{B} n^{n}} \circ \varphi_{f}$.

Assume again that $I$ is centered at 0 with $[-1,1] \subseteq I$ and let $f: I^{n} \rightarrow \mathbb{R}$ be a symmetric quasi-Lovász extension for which there exists $A^{*} \subseteq[n]$ such that $f_{0}\left(\mathbf{1}_{A^{*}}\right) \neq 0$. Then the inner function $\varphi_{f}$ introduced in Theorem 14 is unique. Indeed, by Proposition 5, we have $f_{0}\left(x \mathbf{1}_{A}\right)=\varphi_{f}(x) f_{0}\left(\mathbf{1}_{A}\right)$ for every $x \in I$ and every $A \subseteq[n]$. The function $\varphi_{f}$ is then defined by $\varphi_{f}(x)=\frac{f_{0}\left(x \mathbf{1}_{A^{*}}\right)}{f_{0}\left(\mathbf{1}_{A^{*}}\right)}, x \in I$.

Theorem 15. Assume that $I$ is centered at 0 with $[-1,1] \subseteq I$ and let $f: I^{n} \rightarrow$ $\mathbb{R}$ be a symmetric quasi-Lovász extension, $f=\breve{L} \circ \varphi$. Then there exists $A^{*} \subseteq[n]$ such that $f_{0}\left(\mathbf{1}_{A^{*}}\right) \neq 0$ if and only if there exists $a>0$ such that $\varphi=a \varphi_{f}$ and $\check{L}_{0}=\frac{1}{a}\left(\check{L}_{\left.f\right|^{n}}\right)_{0}$.

Remark 16. If $I=[-1,1]$, then the "nonconstant" assumption and the second condition in assertion $(i)$ of Theorem 14 can be dropped off. 


\section{Acknowledgments}

This research is supported by the internal research project F1R-MTH-PUL09MRDO of the University of Luxembourg.

\section{References}

1. G. Beliakov, A. Pradera, and T. Calvo. Aggregation Functions: A Guide for Practitioners. Studies in Fuziness and Soft Computing. Springer, Berlin, 2007.

2. P. Benvenuti, R. Mesiar, and D. Vivona. Monotone set functions-based integrals. In Handbook of measure theory, Vol. II, pages 1329-1379. North-Holland, Amsterdam, 2002.

3. D. Bouyssou, D. Dubois, H. Prade, and M. Pirlot, editors. Decision-Making Process - Concepts and Methods. ISTE/John Wiley, London, 2009.

4. M. Couceiro and J.-L. Marichal. Axiomatizations of Lovász extensions and symmetric Lovász extensions of pseudo-Boolean functions, Fuzzy sets and Systems 181:1 (2011) 28-38.

5. M. Couceiro and J.-L. Marichal. Axiomatizations of quasi-Lovász extensions of pseudo-Boolean functions, Aequationes Mathematicae 82 (2011) 213-231.

6. L. M. de Campos and M. J. Bolaños. Characterization and comparison of Sugeno and Choquet integrals. Fuzzy Sets and Systems, 52(1):61-67, 1992.

7. M. Grabisch, J.-L. Marichal, R. Mesiar, and E. Pap. Aggregation functions. Encyclopedia of Mathematics and its Applications 127. Cambridge University Press, Cambridge, UK, 2009.

8. M. Grabisch, T. Murofushi, and M. Sugeno, editors. Fuzzy measures and integrals - Theory and applications, volume 40 of Studies in Fuzziness and Soft Computing. Physica-Verlag, Heidelberg, 2000.

9. P. Hammer and S. Rudeanu. Boolean methods in operations research and related areas. Berlin-Heidelberg-New York: Springer-Verlag, 1968.

10. L. Lovász. Submodular functions and convexity. In Mathematical programming, 11th int. Symp., Bonn 1982, 235-25\%. 1983.

11. R. Mesiar and A. Mesiarová-Zemánková. The ordered modular averages. IEEE Trans. Fuzzy Syst., 19(1):42-50, 2011.

12. I. Singer. Extensions of functions of $0-1$ variables and applications to combinatorial optimization. Numer. Funct. Anal. Optimization, 7:23-62, 1984.

13. J. Šipoš. Integral with respect to a pre-measure. Mathematica Slovaca, 29(2):141$155,1979$.

14. D. M. Topkis. Minimizing a submodular function on a lattice. Operations Research, 26(2):305-321, 1978. 